\title{
LUXAÇÃO AGUDA ISOLADA DA ARTICULAÇÃO TIBIOFIBULAR PROXIMAL
}

\section{ACUTE ISOLATED ANTEROLATERAL DISLOCATION OF THE PROXIMAL TIBIOFIBULAR JOINT}

Paulo Roberto de Almeida Silvares', João Paulo Fernandes Guerreiro², Sérgio Swain Müller', Ricardo Violante Pereira ${ }^{3}$, Rodrigo Vannini ${ }^{3}$

\section{RESUMO}

A luxação traumática isolada da articulação tibiofibular proximal é rara. Esta lesão pode não ser reconhecida ou diagnosticada no atendimento inicial. A ausência de suspeita clínica pode causar problemas para o diagnóstico. O diagnóstico necessita de história precisa do mecanismo e sintomas da lesão, avaliação clínica e radiográfica adequada de ambos joelhos. Casos não reconhecidos são fonte de alterações crônicas. O tratamento é feito por redução fechada e imobilização ou, em casos irredutíveis ou instáveis, redução aberta com fixação interna temporária. Um caso raro de luxação tibiofibular proximal isolada em um jogador de basquetebol é relatado para ilustrar essa lesão.

Descritores - Luxação do joelho; Fíbula; Basquetebol; Masculino

\section{ABSTRACT}

The isolated traumatic dislocation of the proximal tibiofibular joint is rare. The injury could go unrecognized or be misdiagnosed at the initial presentation. Lack of clinical suspicion can cause diagnostic problems. The diagnosis requires accurate history of the mechanism and symptoms of the injury, and adequate clinical and radiographic evaluation of the both knees. Overlooked injuries are a source of potentially chronic changes. The treatment is closed reduction and immobilization or, in case of a failed or unstable reduction, open reduction with temporary internal fixation. A rare case of acute isolated anterolateral dislocation of the proximal tibiofibular joint in a basketball player was described to illustrate this injury.

Keywords - Knee dislocation; Fibula; Basketball; Masculine

\section{INTRODUÇÃO}

As lesões na face lateral do joelho são menos frequentes que as mediais. Os ligamentos laterais, inserções tendíneas e a fíbula proximal protegem, mas podem dificultar o exame e o diagnóstico de lesões na região ${ }^{(1-3)}$. Luxação aguda de articulação tibiofibular proximal é de diagnóstico raro e pode passar despercebido nas salas de pronto atendimento e de emergência ${ }^{(4,5)}$. Casos negligenciados ou não tratados podem levar à alterações degenerativas da articulação com dor crônica e até disfunção do nervo fibular ${ }^{(6,7)}$.

\section{ANATOMIA}

A articulação tibiofibular proximal é articulação sinovial entre côndilo tibial lateral e cabeça da fíbula ${ }^{(8,9)}$. Ao menos 10\% da população possui comunicação entre esta articulação e a tibiofemoral, justificando alguns casos de derrame articular discreto no joelho ${ }^{(9,10)}$.

A tibiofibular é articulação naturalmente estável devido à congruência óssea articular, ao envelope musculoligamentar e sua localização posterolateral ${ }^{(7)}$. A orientação da superfície articular pode variar e levar a maior risco de luxação ${ }^{(1)}$. As variantes foram definidas como horizontal

\footnotetext{
1 - Professor Doutor do Departamento de Ortopedia e Traumatologia da Faculdade de Medicina de Botucatu-Unesp.

2 - Médico Residente do Departamento de Ortopedia e Traumatologia da Faculdade de Medicina de Botucatu-Unesp.

3 - Médico do Departamento de Ortopedia e Traumatologia da Faculdade de Medicina de Botucatu-Unesp.
}

Trabalho realizado no Departamento de Ortopedia e Traumatologia da Faculdade de medicina de Botucatu-Unesp.

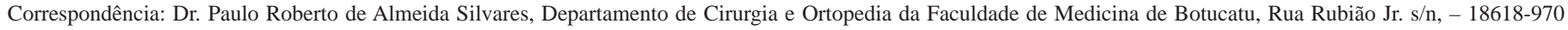

- Botucatu, SP. E-mail: psilvares@fmb.unesp.br 
(até $20^{\circ}$ de inclinação em relação ao plano do solo) ou oblíqua (maior que $20^{\circ}$ inclinação), sendo esta encontrada em $70 \%$ dos pacientes com a lesão ${ }^{(1)}$ (Figura 1).

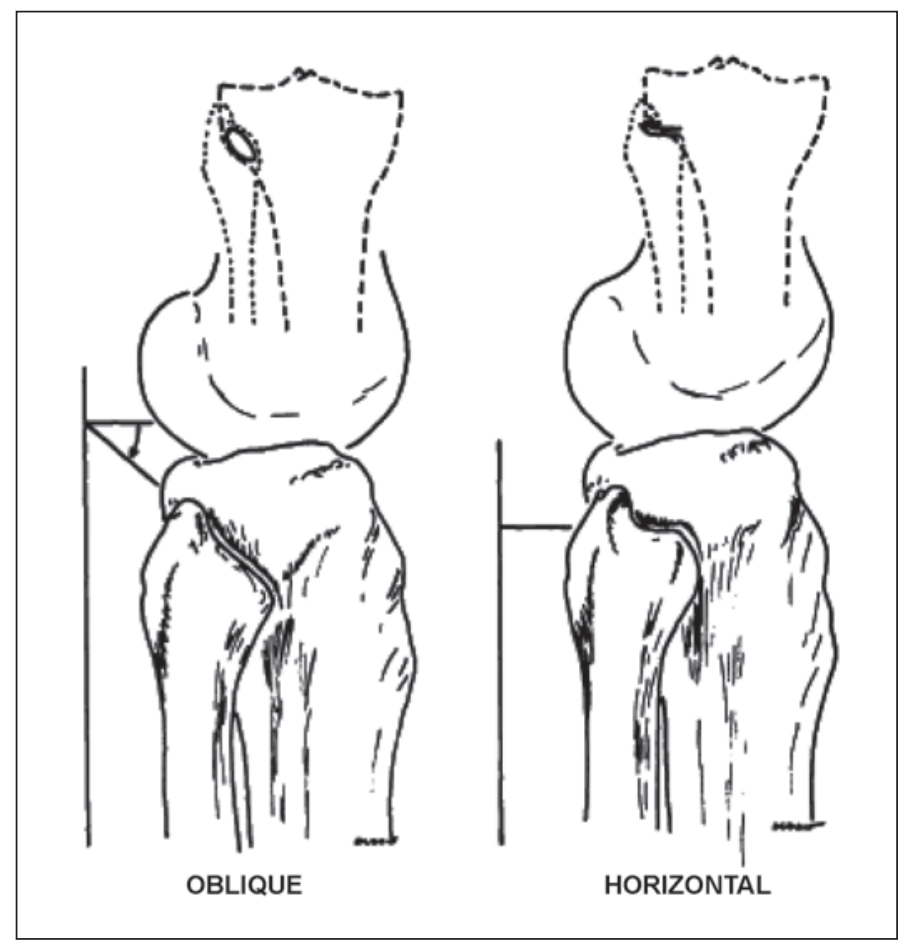

Figura 1 - Anatomia da articulação ${ }^{(1)}$

Os espessamentos capsulares anteriores e posteriores formam os ligamentos tibiofibulares, sendo o tibiofibular anterior o mais resistente ${ }^{(6,9,11)}$. Os estabilizadores adicionais da articulação são o ligamento colateral lateral e o tendão do bíceps femoral (quando o joelho está em extensão), o tendão poplíteo e o ligamento poplíteo fibular ${ }^{(3,9,12)}$. Com o joelho em flexão, a cabeça fibular migra anteriormente enquanto relaxa o ligamento colateral lateral e o tendão do músculo bíceps femoral perdendo estabilidade ${ }^{(1,13-15)}$.

Além da articulação proximal, a tíbia e a fíbula possuem entre si a sindesmose distal, que pode ser lesada no mesmo trauma ${ }^{(9)}$. A função primária da articulação proximal é dissipar forças de torção aplicadas ao tornozelo, dissipar forças de apoio laterais tibiais e transmissão de carga axial ${ }^{(9,14)}$.

\section{QUADRO CLÍNICO}

A história da luxação tibiofibular aguda isolada é associada a graves entorses com inversão e flexão plantar do pé simultânea à flexão do joelho e rotação externa da perna ${ }^{(11,16)}$. A luxação também pode acontecer por traumas diretos em mecanismos de alta energia ${ }^{(1,11,17)}$.
O paciente tem dor espontânea e agravada por inversão, eversão ou dorsoflexão do pé na região lateral inferior do joelho ${ }^{(1,11,16,18-20)}$. É frequente parestesia em território do nervo fibular, mas a paralisia com déficit da dorsoflexão é pouco descrita ${ }^{(2,11,21)}$. Uma proeminência óssea é vista na região da cabeça da fíbula e derrame articular leve pode estar presente ${ }^{(6,12)}$. O joelho tem amplitude de movimento preservada, mas a movimentação causa dor ${ }^{(11,12)}$. A ausência ou discreta presença de equimose e edema são justificadas pela pobre vascularização da área ${ }^{(11,16,19)}$.

O exame da articulação do tornozelo é mandatório para detectar lesões da membrana interóssea e ligamentos da sindesmose ${ }^{(7,22)}$.

\section{QUADRO RADIOLÓGICO}

As radiografias anteroposteriores do joelho normalmente mostram tíbia e fíbula proximal superpostas ${ }^{(23)}$. A comparação entre radiografias de frente e perfil dos dois joelhos ajudam a confirmar o diagnóstico e a localização da cabeça da fíbula ${ }^{(19)}$. A tomografia computadorizada é indicada para melhor estudo da articulação e quando há dúvida diagnóstica ${ }^{(12,24,25)}$.

\section{CLASSIFICAÇÃO}

Quatro tipos, de acordo com o deslocamento, foram descritos por Ogden em 1974(1) (Figura 2).

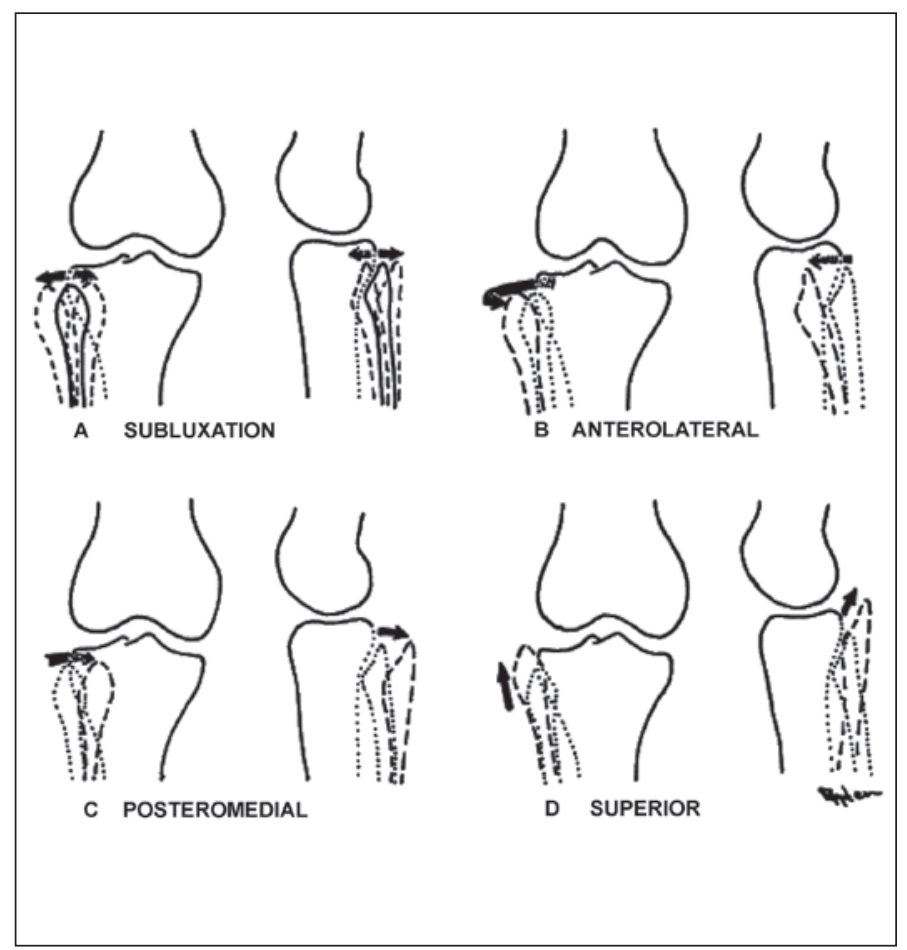

Figura 2 - Classificação de Ogden ${ }^{(1)}$ 
Tipo I - Caracterizado por excessiva mobilidade articular com subluxação multidirecional frequentemente encontrada em pacientes jovens com hipermobilidade articular.

Tipo II - Caracterizado por deslocamento anteroposterior; é o tipo mais comum, ocorrendo em até 85\% dos $\operatorname{casos}^{(1,12,20,26)}$.

Tipo III - Posteromedial; ocorre em 10\% dos casos e é mais associado ao trauma direto na cabeça da fíbula ${ }^{(12,17)}$. Geralmente é mais instável após redução fechada inicial, o que dificulta o tratamento conservador ${ }^{(1,16)}$.

Tipo IV - É um deslocamento superior da cabeça da fíbula associado à fratura do colo da fíbula ou trauma de grande energia no tornozelo com grave lesão da sindesmose tibiofibular ${ }^{(11,16,26)}$.

\section{TRATAMENTO}

A princípio a luxação aguda deve ser reduzida de maneira incruenta com anestesia local ou sedação endovenosa ${ }^{(12,16,27,28)}$.

A manobra exige pressão direta na cabeça da fíbula, contrária ao deslocamento, com flexão de aproximadamente $90^{\circ}$ do joelho para relaxar o ligamento colateral lateral e o tendão do bíceps femoral ${ }^{(1,7,11,16,19,17,29)}$. Ao se manter o pé rodado externamente, evertido e dorsofletido, teoricamente, relaxam-se os músculos fibulares, extensor longo do hálux e dos dedos, e facilitaria a redução ${ }^{(1,2,4,6)}$.

Após a redução e comprovada a estabilidade, a maioria dos autores indica três semanas de imobilização do joelho em leve flexão e tornozelo em $90^{\circ}$ de dorsoflexão. Após a retirada da imobilização inicia-se movimentação sem carga por mais três semanas ${ }^{(6,10-12,30)}$.

Os casos não redutíveis ou instáveis após a redução necessitam redução aberta e fixação interna provisória assim que possível ${ }^{(7,11,20-22,31)}$. Podem ser usados fios de Kirschner ou parafusos que devem ser retirados entre seis e 12 semanas ou pinos bioabsorvíveis ${ }^{(4,11,19,21,32-34)}$.

\section{RELATO DE CASO}

Paciente masculino, 20 anos, estudante e membro de uma equipe universitária de basquetebol. Deu entrada no pronto-socorro queixando-se que, após entorse de tornozelo associada à rotação lateral da perna em relação ao joelho direito, sentiu forte dor, a qual permaneceu constante, na face lateral do joelho direito.
Ao exame físico apresentava cabeça da fíbula direita protuberante, com discreto edema (Figura 3), sem derrame articular ou equimose. Dor à palpação da fíbula proximal e amplitude de movimento preservada, apesar de apresentar exacerbação da dor durante o exame. Havia ainda discreta dor à palpação da região da sindesmose tibiofibular do tornozelo, sem edema local. Ausência de sinais clínicos de lesão do nervo fibular.

Ao exame radiográfico de frente e perfil dos joelhos (Figura 4) notou-se deslocamento anterolateral da fíbula proximal direita comparando-se com a articulação à esquerda.

Tentada redução frustrada da luxação na sala do pronto-socorro após anestesia local com lidocaína 1\%, mantendo-se o joelho fletido e fazendo-se pressão sobre a cabeça da fíbula na direção posterior (Figura 5). Após, encaminhado ao centro cirúrgico onde, após anestesia raquidiana subaracnoidea e a mesma manobra citada, foi obtida redução anatômica confirmada por novas radiografias (Figura 6).

Graças à estabilidade obtida depois da redução e após estudo da articulação do tornozelo, foi realizado o tratamento com imobilização do joelho e tornozelo direito por três semanas, seguido por mais três semanas com movimentação das articulações protegendo-as de carga. Após seis semanas teve retorno à carga e exercícios para fortalecimento muscular com sucesso.

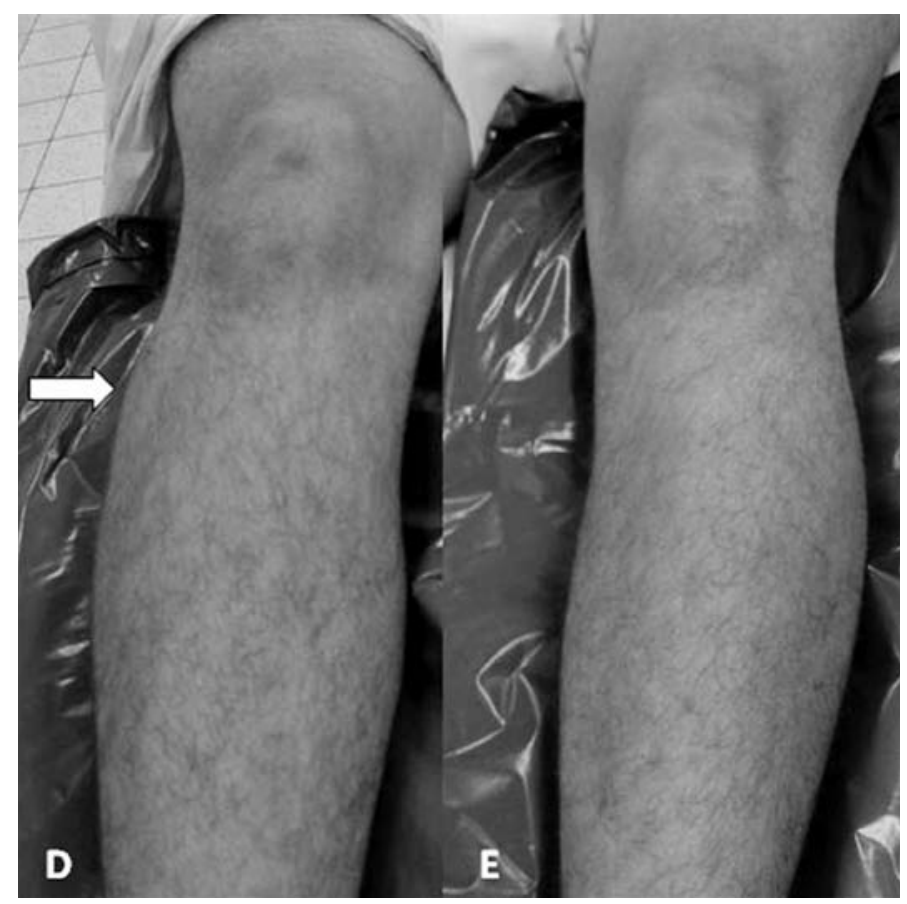

Figura 3 - Joelho direito e esquerdo antes da redução 


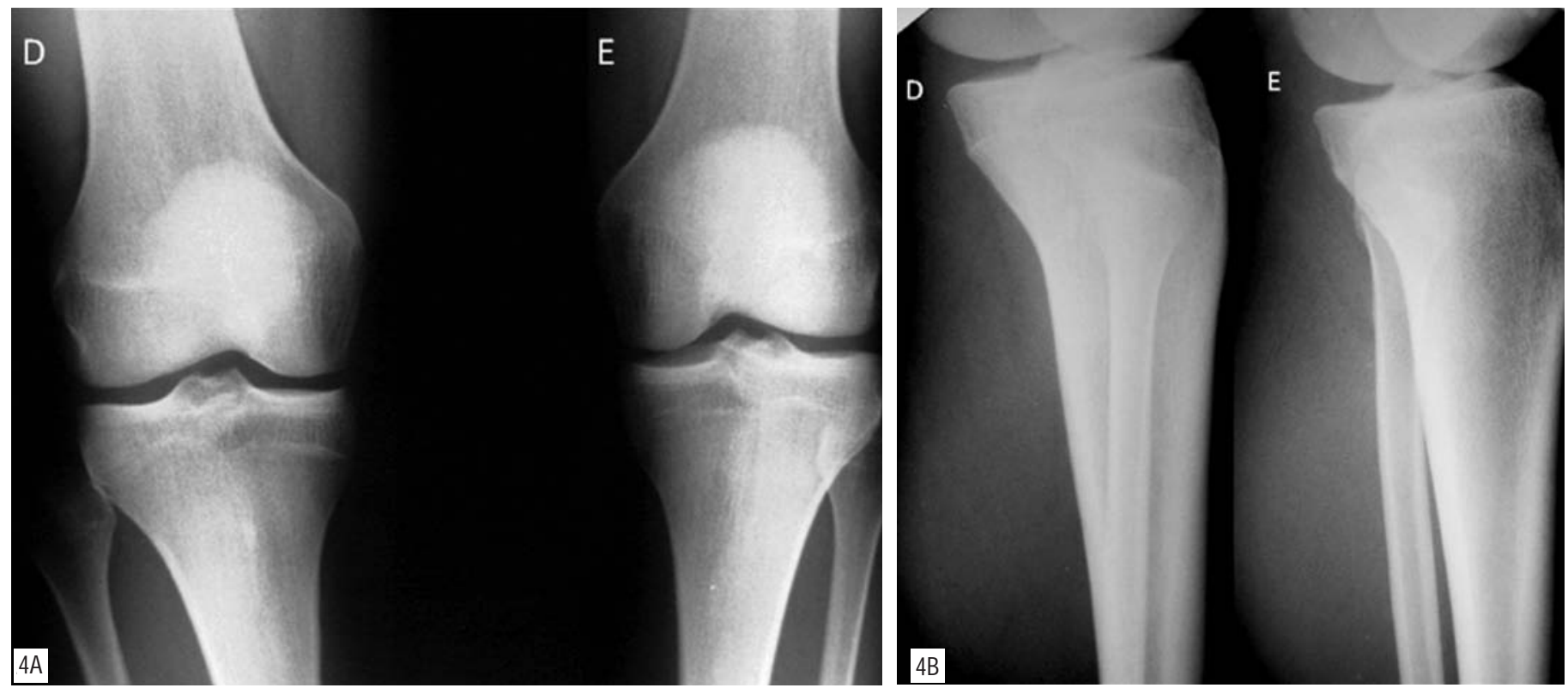

Figura 4 - A) Radiografia AP: joelho direito e joelho esquerdo antes da redução. B) Radiografia perfil: joelho direito e joelho esquerdo antes da redução

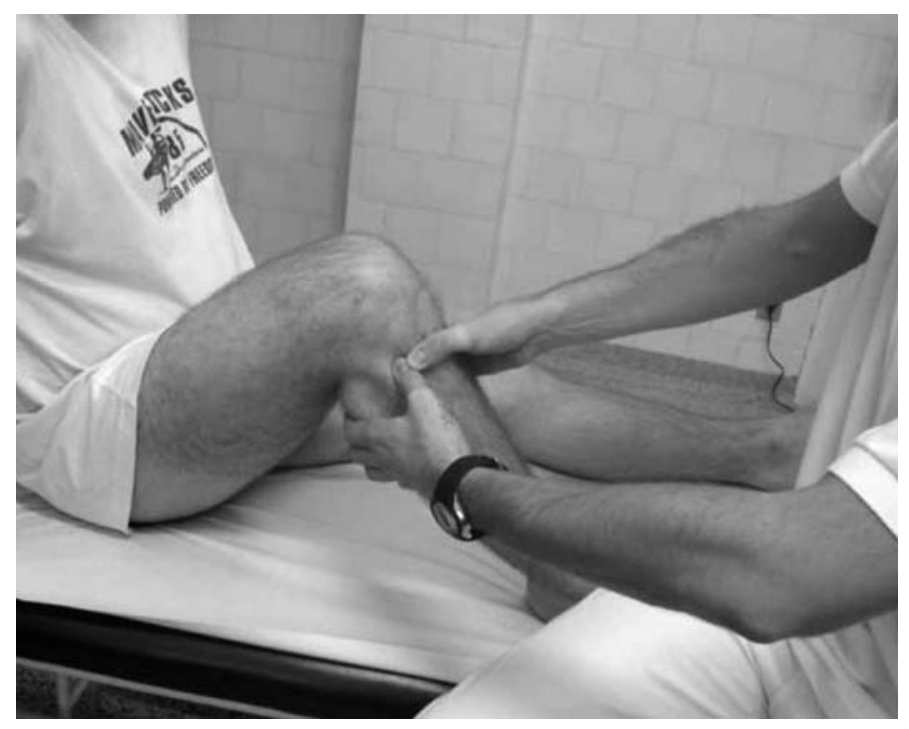

Figura 5 - Manobra de redução realizada

\section{DISCUSSÃO}

Desde a primeira descrição de luxação tibiofibular proximal por Nelaton ${ }^{(35)}$, em 1874, e o clássico trabalho de Ogden ${ }^{(1)}, 100$ anos depois, sabe-se que esta lesão existe, é rara, e mais ainda de forma isolada.

Casos desde o final do século XIX até os dias atuais são relatados isoladamente por todo o mundo(3,4,6,12,36-38). A maior revisão já realizada, de 1974, encontrou apenas 108 casos na literatura ${ }^{(1)}$.

Devido à raridade desta lesão, deve-se ficar atento

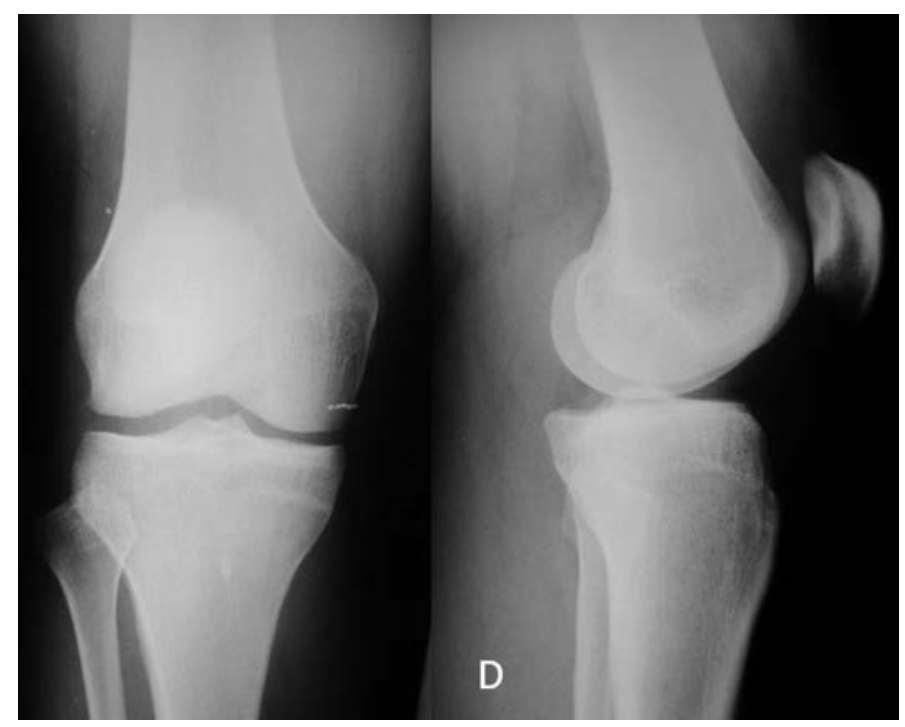

Figura 6 - Radiografia AP e perfil após a redução

para realizar o diagnóstico ${ }^{(6,12)}$. A radiografia isolada do joelho em questão geralmente não sugere a lesão ${ }^{(12,19,23-25)}$. Por essas razões, os poucos relatos da literatura podem não corresponder à realidade, e luxações tibiofibulares proximais podem estar passando despercebidas nos pronto-socorros de todo o mundo ${ }^{(1,6)}$.

O conhecimento da possibilidade da lesão, a história clínica bem realizada, o exame físico adequado e a radiografia comparativa dos joelhos, nos casos suspeitos, são o caminho para o diagnóstico no momento adequado, facilitando o tratamento e melhorando o prognóstico ${ }^{(1,5,6,12,19,29)}$. 


\section{REFERÊNCIAS}

1. Ogden JA. Subluxation and dislocation of the proximal tibiofibular joint. J Bone Joint Surg Am. 1974;56(1):145-54.

2. Ogden JA. Subluxation of the proximal tibiofibular joint. Clin Orthop Relat Res. 1974;(101):192-7.

3. Ellis C. A case of isolated proximal tibiofibular joint dislocation while snowboarding. Emerg Med J. 2003;20:563-4.

4. Crothers OD, Johnson JT. Isolated acute dislocation of the proximal tibiofibular joint. Case report. J Bone Joint Surg Am. 1973;55(1):181-3.

5. Love JN. Isolated anterolateral proximal fibular head dislocation. Ann Emerg Med. 1992;21(6):757-9.

6. Iosifidis MI, Giannoulis I, Tsarouhas A, Traios S. Isolated acute dislocation of the proximal tibiofibular joint. Orthopedics. 2008;31(6):605.

7. Aladin A, Lam KS, Szypryt EP. The importance of early diagnosis in the management of proximal tibiofibular dislocation: a 9- and 5-year follow-up of a bilateral case. Knee. 2002;9(3):233-6.

8. Jobe CM, Wright M. Anatomy of the knee. In: Fu FH, Harner CD, Vince KG, editors. Knee surgery. Baltimore: Williams \& Wilkins; 1994. p. 25-6.

9. Ogden JA. The anatomy and function of the proximal tibiofibular joint. Clin Orthop Relat Res. 1974;(101):186-91.

10. Bozkurt M, Yilmaz E, Atlihan D, Tekdemir I, Havitcioglu H, Gunal I. The proximal tibiofibular joint: an anatomic study. Clin Orthop Relat Res. 2003;(406):136-40.

11. Sekiya JK, Kuhn JE. Instability of the proximal tibiofibular joint. J Am Acad Orthop Surg. 2003;11(2):120-8.

12. Van Seymortier P, Ryckaert A, Verdonk P, Almqvist KF, Verdonk R. Traumatic proximal tibiofibular dislocation. Am J Sports Med. 2008;36(4):793-8.

13. Luscombe KL, Maffuli N. Stabilization of the superior tibiofibular joint. Techn Knee Surg. 2005;4:126-30.

14. Moorman CT 3rd, LaPrade RF. Anatomy and biomechanics of the posterolateral corner of the knee. J Knee Surg. 2005;18(2):137-45.

15. Watanabe $\mathrm{Y}$, Moriya H, Takahashi K, Yamagata M, Sonoda M, Shimada Y, et al. Functional anatomy of the posterolateral structures of the knee. Arthroscopy. 1993;9(1):57-62.

16. Thomason PA, Linson MA. Isolated dislocation of the proximal tibiofibular joint. J Trauma. 1986;26(2):192-5.

17. Horan J, Quin G. Proximal tibiofibular dislocation. Emerg Med J. 2006;23(5):e33-4.

18. Veth RP, Klasen HJ, Kingma LM. Traumatic instability of the proximal tibiofibular joint. Injury. 1981;13(2):159-64.

19. Turco VJ, Spinella AJ. Anterolateral dislocation of the head of the fibula in sports. Am J Sports Med. 1985;13(4):209-15.

20. Falkenberg $\mathrm{P}, \mathrm{Nygaard} \mathrm{H}$. Isolated anterior dislocation of the proximal tibiofibular joint. J Bone Joint Surg Br. 1983;65(3):310-1.
21. Parkes JC 2nd, Zelko RR. Isolated acute dislocation of the proximal tibiofibular joint. Case report. J Bone Joint Surg Am. 1973;55(1):177-83.

22. Levy BA, Vogt KJ, Herrera DA, Cole PA. Maisonneuve fracture equivalent with proximal tibiofibular dislocation. A case report and literature review. J Bone Joint Surg Am. 2004;88(5):1111-6.

23. Rockwood CA, Green DP. Fractures in adults. Philadelphia: Linppincott. 5th ed. 2001; p. 1928-31.

24. Keogh P, Masterson E, Murphy B, McCoy CT, Gibney RG, Kelly E. The role of radiography and computed tomography in the diagnosis of acute dislocation of the proximal tibiofibular joint. Br J Radiol. 1993;66(782):108-11.

25. Voglino JA, Denton JR. Acute traumatic proximal tibiofibular dislocation confirmed by computed tomography. Orthopedics. 1999;22(2):255-58.

26. Molitor PJ, Dandy DJ. Permanent anterior dislocation of the proximal tibiofibular joint. J Bone Joint Surg Br. 1989;71(2):240-1.

27. Resnick D, Newell JD, Guerra J Jr, Danzig LA, Niwayama G, Goergen TG. Proximal tibiofibular joint: anatomic-pathologic-radiographic correlation. AJR Am J Roentgenol. 1978;131(1):133-8.

28. Miettinen $\mathrm{H}$, Kettunen J, Vaatainen U. Dislocation of the proximal tibiofibular joint. A new method for fixation. Arch Orthop Trauma Surg. 1999;119(5-6):358-9.

29. Petter A, Davidson J. An unusual knee injury: isolated tibiofibular dislocation. Emerg Med Australas. 2004;16(2):172-3.

30. Semonian RH, Denlinger PM, Duggan RJ. Proximal tibiofibular subluxation relationship to lateral knee pain: a review of proximal tibiofibular joint pathologies. J Orthop Sports Phys Ther. 1995;21(5):248-57.

31. Falkenberg $P$, Nygaard $\mathrm{H}$. Isolated anterior dislocation of the proximal tibiofibular joint. J Bone Joint Surg Br. 1983;65(3):310-1.

32. Parkes JC 2nd, Zelko RR. Isolated acute dislocation of the proximal tibiofibular joint. Case report. J Bone Joint Surg Am. 1973;55(1):177-83.

33. Rajkumar P, Schmiteen GF. A new surgical treatment of an acute dislocation of the proximal tibiofibular joint. Int J Clin Pract. 2002;56(7);556-7.

34. Van den Bekerom MP, Weir A, van der Flier RE. Surgical stabilization of the proximal tibiofibular joint using temporary fixation: a technical note. Acta Orthop Belg. 2004;70(6):604-8.

35. Nelaton A. Elements de pathologic chirurgicale. 2a. ed. Paris: Librairie Germer Ballière; 1874. p. 282.

36. Fishbourne JE. Dislocation ofthe head ofthe fibulaforwards and "Upwards". British Med J. 1887;1:617.

37. Barabo W. Uber eine isolierte Luxation des linken Wadenbeinkopfchens nach hinten. Zentralbl F Chir. 1911;38:268-70.

38. Auffay Y. Un nouveau cas d'une luxation rare: la luxation antérieure isolée de l'extrémité supérieure du pérone. Soc Nat Med et des Sciences Médicales, Lyon, 1965. 